\title{
Tetrahedral rigid core antenna chromophores bearing
}

\section{bay-substituted perylenediimides}

\author{
Mykhaylo Myahkostupov and Felix N. Castellano* \\ Department of Chemistry, North Carolina State University, Raleigh, NC 27695-8204, USA
}

*To whom correspondence should be addressed. Email: fncastel@ncsu.edu; Phone: (919) 515-3021;

Fax: (919) 515-8920 


\begin{abstract}
Two new representative methane- and adamantane-centered "antenna" tetramers bearing baysubstituted $\pi$-conjugated phenylethynyl-perylenediimides (PDICCPh) as chromophoric subunits, tetrakis-[1-(4-ethynylphenyl)-N,N'-bis(1-hexylheptyl)-perylene-3,4:9,10-tetracarboxylic diimide]methane (1) and tetrakis-1,3,5,7-[1-(4-ethynylphenyl)-N,N'-bis(1-hexylheptyl)-perylene-3,4:9,10tetracarboxylic diimide]-adamantane (2), have been synthesized and their structural aspects have been thoroughly investigated by NMR spectroscopy. These PDI tetramers (1 and 2) represent the first successful example of incorporating the bay-substituted phenylethynyl-perylenediimides into the large rigid core tetrahedral frameworks. In these PDI tetramers, dynamic NMR experiments revealed the existence of perylene-centered conformational dynamic equilibrium $\left(\Delta G^{\ddagger}=15-17 \mathrm{kcal} / \mathrm{mol}\right)$, the primary cause of the observed spectral broadening in conventional ${ }^{1} \mathrm{H}$ NMR spectra (295 K). In addition, PDI tetramers 1 and 2 were found to possess exceptional (photo)chemical stability, and their corresponding photophysical properties $\left(\varepsilon_{\max } \sim 180,000 ; \tau_{\mathrm{FL}}=6.9 \mathrm{~ns} ; \Phi_{\mathrm{FL}} \sim 60 \%\right)$ make them viable candidates for various photonic applications and are in good agreement with other related multichromophoric PDI-based systems.
\end{abstract}

Keywords: perylenediimide; rigid core chromophores; Sonogashira coupling; dynamic NMR; conformational exchange

\title{
1. Introduction
}

Over the years, perylenediimide-based (PDI) chromophoric systems have been the subject of immense research efforts thanks to their unique combination of chemical, photochemical, and photophysical properties. ${ }^{1-6}$ Multichromophoric systems incorporating a broad spectrum of PDI molecules proved to be excellent models for exploring fundamental and applied aspects of diverse photonic, electronic, and photovoltaic materials. ${ }^{7-16}$ In addition, various carefully designed PDI-based molecular compositions have been utilized as probes of photoinduced ultrafast processes (e.g., those 
occurring in natural photosynthetic systems), thus leading to a better understanding of underlying quantum coherence, excitation energy flow and excitonic coupling effects. ${ }^{17-25}$ Similarly, molecular PDI acceptor chromophores have been exploited in numerous upconversion photochemistry compositions. $^{10,12,26}$

The synthetic accessibility of PDI functionalization chemistry (either at the imide position or in the bay region) allows one to construct multichromophoric assemblies with a desired set of functional properties. ${ }^{8,27-29}$ The goal of the present work is to utilize the highly emissive PDI chromophores with extended $\pi$-conjugation in the bay region as building blocks to construct three-dimensional, electronically coupled molecular systems (tetrameric “antennas") that would exhibit relatively strong intramolecular electronic interactions and desirable photophysics as a result of being held together by a tetrahedral rigid core. In this particular case, the extent of intramolecular electronic coupling can be manipulated by varying the size of the rigid core: methane vs. adamantane. In addition, as prototype photochemically robust antenna chromophores and potentially viable alternatives to fullerenes, these PDI tetramers may be of particular interest for photovoltaic applications as well as in photochemical upconversion. ${ }^{7,30-32}$ Noteworthy, the rigid cores of this nature have been extensively explored in a variety of visible light sensitizers (both pure organic and transition metal-based) designed for the covalent surface functionalization of wide bandgap semiconductor nanomaterials (e.g., $\left.\mathrm{TiO}_{2}, \mathrm{ZnO}\right){ }^{33-37}$

PDI-based molecular systems are also well known to display solvent-dependent aggregation behavior, thus making them perfect candidates for probing various energy and electron transfer pathways in both aggregated and non-aggregated forms. ${ }^{38-42}$ However, we note that any detailed discussion of the associated excited state photochemistry of synthesized PDI tetramers is well beyond the scope of the present work and will be the subject of a separate in-depth contribution. To date, there is only a handful of (photophysical) studies on PDI-containing tetrahedral assemblies and, in most cases, these PDI architectures have been synthesized utilizing a relatively straightforward imide condensation chemistry between the amine-functionalized rigid core(s) and the corresponding perylene dianhydride(s). ${ }^{1,41,43}$ Moreover, the successful examples of tetrameric structures incorporating the bay- 
substituted PDIs have been quite scarce in the literature and the few available reports are mainly based on studying the properties of phenyl-PDI-containing structures. ${ }^{44,45}$ In our case, it is expected that the incorporation of phenylethynyl-perylenediimides $(\mathrm{PDICCPh})$ bearing extended $\pi$-conjugation into tetrahedral rigid core structures would result in the substantial enhancement of their corresponding lightharvesting properties in a broader spectral window and thus may be advantageous in various photonic applications as compared to other related PDI-based oligomeric structures.

In this manuscript, we report the synthesis, NMR structural characterization and photophysical properties of two representative methane- and adamantane-centered tetramers bearing bay-substituted $\pi$ conjugated phenylethynyl-perylenediimides (PDICCPh) as chromophoric subunits that also incorporate solubilizing 1-hexylheptyl functionalities at the imide positions, tetrakis-[1-(4-ethynylphenyl)-N,N'bis(1-hexylheptyl)-perylene-3,4:9,10-tetracarboxylic diimide]-methane (1) and tetrakis-1,3,5,7-[1-(4ethynylphenyl)-N,N'-bis(1-hexylheptyl)-perylene-3,4:9,10-tetracarboxylic diimide]-adamantane (2). To delineate the electronic and structural effects associated with the tetrahedral assembly of four PDICCPh subunits into a large tetrameric framework, 1 and 2 are compared to the model monomer 3 , a single structural subunit of each target PDI tetramer. The corresponding chemical structures of the newly synthesized PDI tetramers 1 and 2, as well as the model PDI monomer 3 are presented in Figure 1. To the best of our knowledge, this work represents the first example when the bay-substituted phenylethynyl-perylenediimides ( $\mathrm{PDICCPh}$ ) have been successfully incorporated into the large rigid core tetrameric frameworks. We also note that the adequate interpretation of the associated NMR structural characterization data of these large PDI assemblies can be quite challenging due to the substantial broadening of ${ }^{1} \mathrm{H}$ resonances (at RT) and is oftentimes overlooked in the literature. In some cases, it was argued that oligomeric PDIs exhibit such behavior as a result of aggregation-induced effects occurring at the typical NMR sample concentrations (mM range). ${ }^{46}$ To fully address these questions, dynamic NMR experiments as well as concentration-dependent NMR studies have been performed to understand the origin of observed spectral broadening associated with single temperature $(295 \mathrm{~K}){ }^{1} \mathrm{H}$ NMR experiments in the target PDI tetramers 1 and $2 .{ }^{29,47,48}$ 


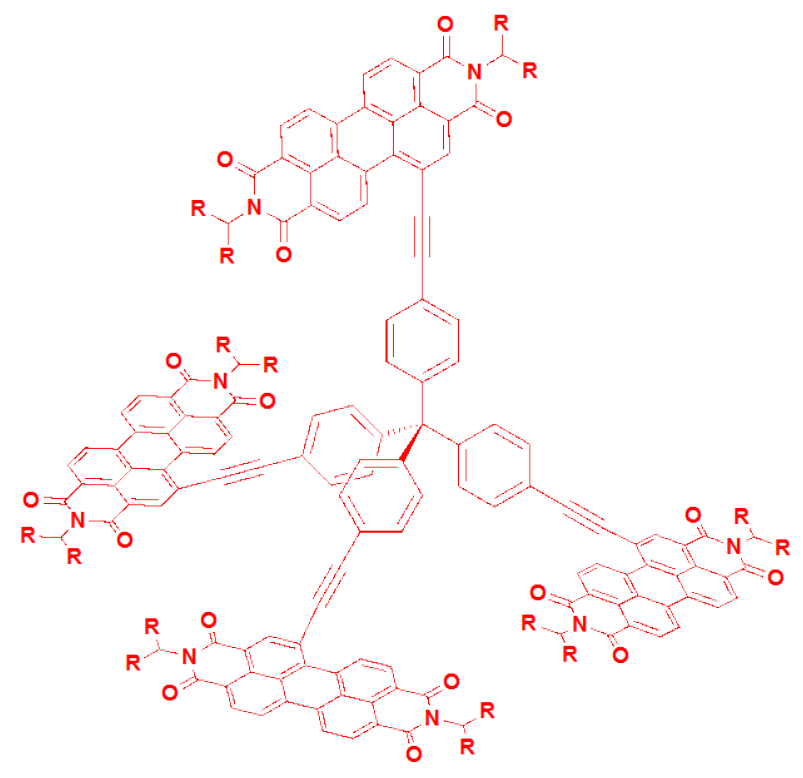

1

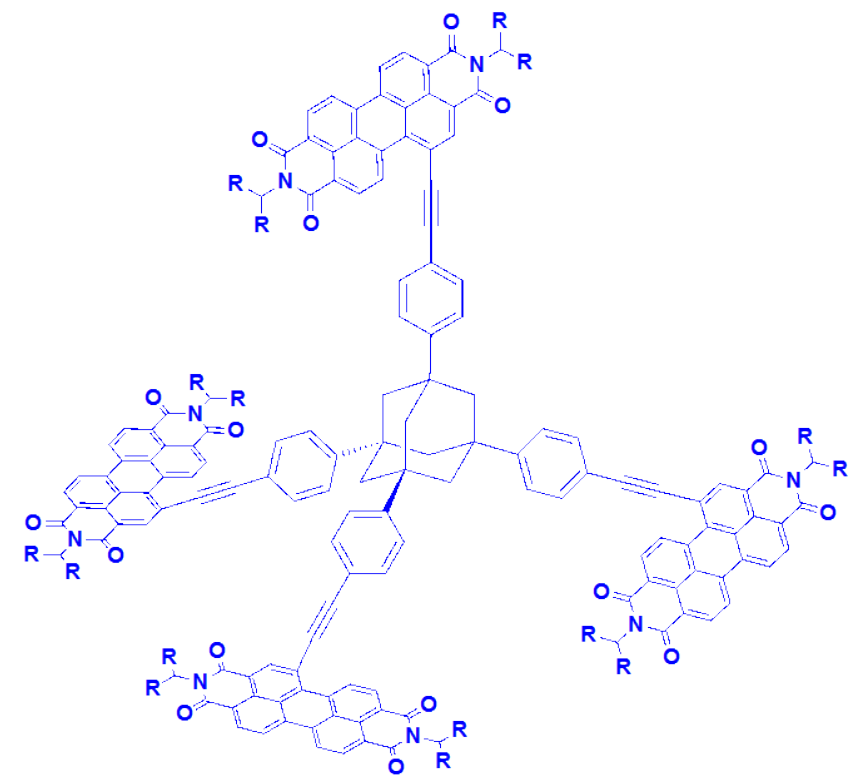

2

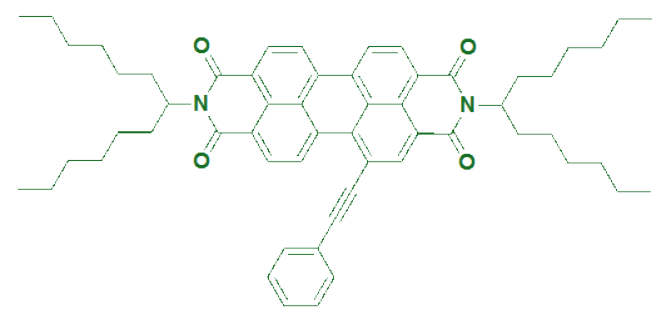

3

Figure 1. Structures of tetrahedral rigid core perylenediimide (PDI) tetramers and a model monomer: methane-centered PDI tetramer 1 (red), adamantane-centered PDI tetramer 2 (blue), and PDICCPh 3 (green). $\boldsymbol{R}$ denotes a hexyl $\left(\mathrm{C}_{6} \mathrm{H}_{13}\right)$ group.

\section{Results and discussion}

2.1. Synthesis. The monomeric PDI precursors (PDI and PDIBr) were synthesized via modified literature procedures and the representative synthetic methodology is presented as Supplementary Data in Scheme S1. ${ }^{49,50}$ In general, the symmetric 1-hexylheptylamine was obtained in $95 \%$ yield via a facile reductive amination of commercially available dihexylketone in the presence of $\mathrm{NaBH}_{3} \mathrm{CN}$. In the next step, 1-hexylheptylamine was condensed under ambient conditions with commercially available 3,4,9,10-perylene-tetracarboxylic dianhydride (PDA) to obtain the corresponding perylenediimide (PDI) in a nearly quantitative yield (95\%). PDI was then selectively brominated in the 1-position of its bay region with elemental $\mathrm{Br}_{2}$ to afford 1-bromoperylenediimide (PDIBr) in 65\% yield. Subsequently, 
PDIBr was used as a departure point to gain access to the target PDI tetramers 1 and 2 (Schemes 1 and 2), as well as the model monomer 3 (Scheme S1). Monomer 3 was obtained via a standard Sonogashira cross-coupling protocol of PDIBr with phenylacetylene $(\mathrm{PhCCH})$ in $75 \%$ yield (44\% overall) as a dark red solid (see Supplementary data). ${ }^{51-53}$

2.1.1. PDI tetramer 1. The synthetic transformation sequence towards PDI tetramer $\mathbf{1}$ is shown in Scheme 1 . The tetrahedral methane-centered core with $4 \pi$-conjugated phenylethynyl $(\mathrm{CCPh})$ arms (M4) was constructed starting from commercially available tetraphenylmethane (M1). ${ }^{54}$ The below described approach is advantageous over other possible synthetic strategies as it allows one to avoid using perylenediimide-acetylene $(\mathrm{PDICCH})$ in final Sonogashira cross-coupling reaction steps and thus circumvents any possible formation of undesirable PDI homodimeric products via Glazer-type coupling. ${ }^{55}$

In the first step, tetraphenylmethane (M1) was conveniently brominated with elemental $\mathrm{Br}_{2}$ under ambient conditions to yield tetrakis(4-bromophenyl)methane (M2) in 80\%. In the next step, M2 was Sonogashira cross-coupled in a facile fashion with trimethylsilylacetylene (TMSA) using a standard reaction protocol to obtain tetrakis(4-trimethylsilylethynylphenyl)methane (M3) in $82 \%$ yield. M3 was then deprotected with aqueous $\mathrm{K}_{2} \mathrm{CO}_{3}$; however, the removal of TMS group did not proceed very cleanly and typical product yields of tetrakis(4-ethynylphenyl)methane (M4) were around 50\%. At this point, any other alternative deprotection strategies, such as treating with TBAF, have not been explored. In the final step, tetrakis(4-ethynylphenyl)methane core (M4) was Sonogashira cross-coupled with slight excess of PDIBr (4.4 eq.) to ensure the complete conversion into the tetrasubstituted target PDI tetramer 1. Afterwards, 1 was carefully purified via several rounds of recrystallization (liquid diffusion) from $\mathrm{MeOH} / \mathrm{DCM}=3 / 1$ and isolated in $64 \%$ yield $(25 \%$ overall) as a dark red solid. We note that purification of PDI tetramers by column chromatography (silica gel) can be challenging as we previously experienced uncontrollable product degradation with other related PDI-based oligomeric compounds. 


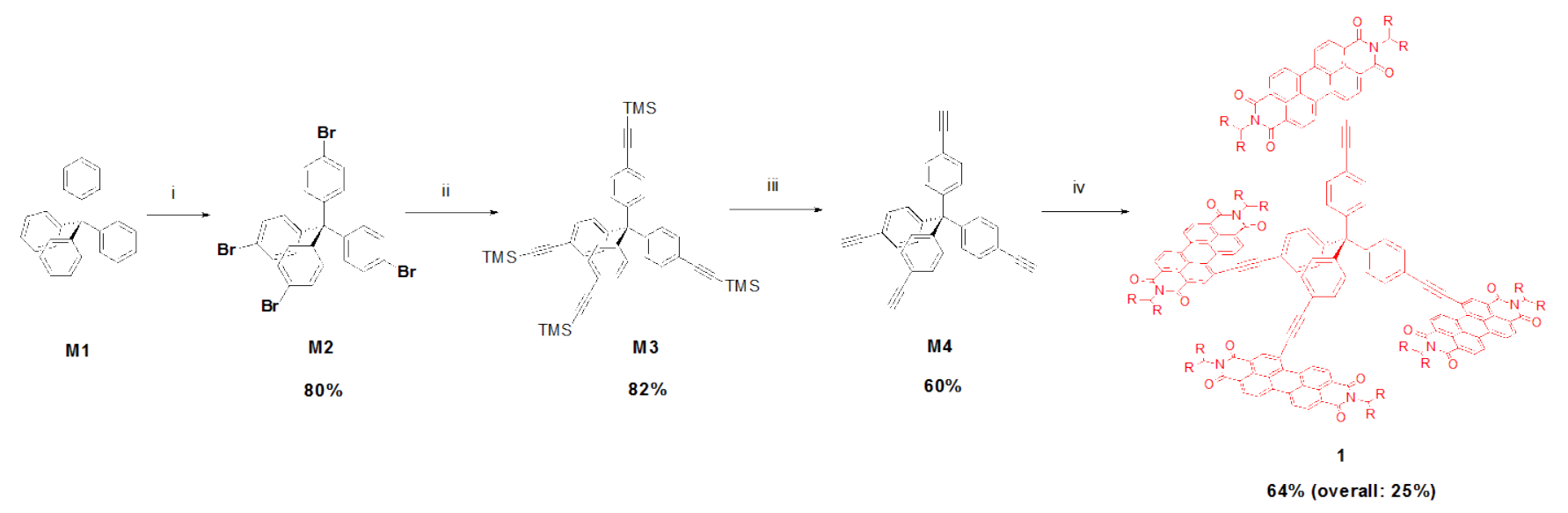

Scheme 1. Synthetic route towards PDI tetramer 1 . Reagents and conditions: (i) $\mathrm{Br}_{2} ; \mathrm{rt} ; 1 \mathrm{~h}$; (ii) Sonogashira: TMSA; $\mathrm{Pd}\left(\mathrm{PPh}_{3}\right)_{4}$; CuI; $\left({ }_{2} \mathrm{Pr}\right)_{2} \mathrm{NH} ; \mathrm{DCM}$ (anh.); $60{ }^{0} \mathrm{C} ; 24 \mathrm{~h}$; (iii) $\mathrm{K}_{2} \mathrm{CO}_{3}$ (aq.); $\mathrm{CHCl}_{3} / \mathrm{MeOH} ; \mathrm{rt} ; 24$ h; (iv) Sonogashira: $\mathrm{PDIBr}$ (4.4 eq.); $\mathrm{Pd}\left(\mathrm{PPh}_{3}\right)_{4} ; \mathrm{CuI} ;\left(\right.$ IPr) ${ }_{2} \mathrm{NH} ; \mathrm{DCM}($ anh.); 65 ${ }^{0} \mathrm{C} ; 48$ h. $\boldsymbol{R}$ denotes a hexyl $\left(\mathrm{C}_{6} \mathrm{H}_{13}\right)$ group.

2.1.2. PDI tetramer 2. The synthetic route towards PDI tetramer 2 is presented in Scheme 2. Similar to PDI tetramer 1, we started from constructing the tetrahedral adamantane-centered core bearing $4 \pi$-conjugated phenylethynyl $(\mathrm{CCPh})$ arms $(\mathbf{A 5})$. In the first step, 1,3,5,7tetraphenyladamantane (A2) was obtained as an insoluble white solid in 95\% yield from commercially available 1-bromoadamantane (A1) following the procedure developed by Reichert and Mathias. ${ }^{56}$ In the next step, A2 was converted into 1,3,5,7-tetrakis(4-iodophenyl)adamantane (A3) via treatment with elemental $\mathrm{I}_{2}$ and [bis(trifluoroacetoxy)iodo]benzene (BFIB) in chloroform. ${ }^{56}$ Due to the intrinsically heterogeneous nature of this reaction, in our hands the yields of $\mathbf{A 3}$ never exceeded 35\% and usually required extended reaction times and moderate heating ( 4 days, $\left.40{ }^{0} \mathrm{C}\right)$. Apparently, this reaction step appears to be limiting in terms of overall yield of PDI tetramer 2. Alternatively, we thought of overcoming this issue by synthesizing 1,3,5,7-tetrakis(4-bromophenyl)adamantane; however, all our attempts (reactions with either elemental $\mathrm{Br}_{2}$ or $\mathrm{Fe}$ powder/Br${ }_{2}$ ) always produced complex, difficult to separate mixtures of products. ${ }^{57}$ In the next step, 1,3,5,7-tetrakis(4-iodophenyl)adamantane (A3) was Sonogashira cross-coupled with trimethylsilylacetylene (TMSA) to obtain 1,3,5,7-tetrakis(4trimethylsilylethynylphenyl)adamantane (A4) in $80 \%$ yield. Subsequently, A4 was deprotected with aqueous $\mathrm{K}_{2} \mathrm{CO}_{3}$. As discussed in the previous section, the deprotection of TMS group typically 
produced the yields of 1,3,5,7-tetrakis(4-ethynylphenyl)adamantane (A5) around 50\%. In the final step, 1,3,5,7-tetrakis(4-ethynylphenyl)adamantane core A5 was Sonogashira cross-coupled with slight excess of PDIBr (4.4 eq.) to yield the target PDI tetramer 2. The purification of 2 was carried in a fashion similar to 1 , thus affording the PDI tetramer 2 in $75 \%$ yield (10\% overall) as a dark red solid.

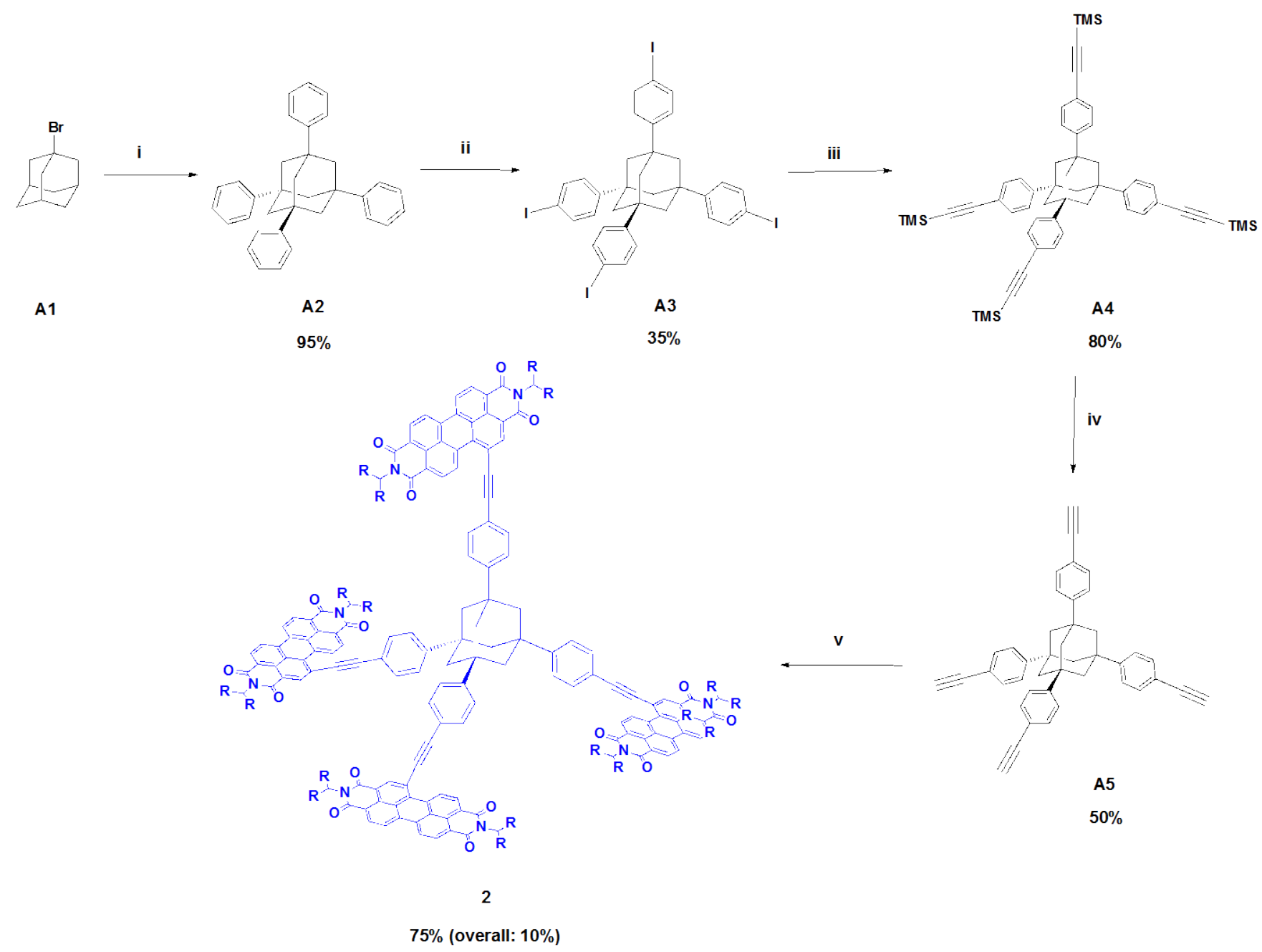

Scheme 2. Synthetic route towards PDI tetramer 2. Reagents and conditions: (i) benzene (anh.); $\mathrm{AlCl}_{3}$; tert-BuBr; reflux; 3 h; (ii) $\mathrm{I}_{2}$; $\mathrm{BFIB} ; \mathrm{CHCl}_{3} ; 40{ }^{0} \mathrm{C} ; 4$ days; (iii) Sonogashira: TMSA; $\mathrm{Pd}\left(\mathrm{PPh}_{3}\right)_{4} ; \mathrm{CuI}$; $\left({ }_{i} \mathrm{Pr}\right)_{2} \mathrm{NH}$; DCM (anh.); $60{ }^{0} \mathrm{C} ; 24 \mathrm{~h}$; (iv) $\mathrm{K}_{2} \mathrm{CO}_{3}$ (aq.); $\mathrm{CHCl}_{3} / \mathrm{MeOH} ; 40{ }^{0} \mathrm{C} ; 24 \mathrm{~h}$; (v) Sonogashira: PDIBr (4.4 eq.); $\mathrm{Pd}\left(\mathrm{PPh}_{3}\right)_{4}$; CuI; ( $\left.{ }_{2} \mathrm{Pr}\right)_{2} \mathrm{NH}$; DCM (anh.); $65{ }^{0} \mathrm{C} ; 48$ h. $\boldsymbol{R}$ denotes a hexyl $\left(\mathrm{C}_{6} \mathrm{H}_{13}\right)$ group.

2.2. NMR structural characterization. Representative ${ }^{1} \mathrm{H}$ NMR spectra (measured in $\mathrm{CDCl}_{3}$ ) of PDIBr, PDI tetramers 1 and 2, and PDI monomer 3 are displayed in Figure 2. The corresponding atomlabeling scheme is presented in Figure 3. The assignment of ${ }^{1} \mathrm{H}$ chemical shifts was based on the comprehensive analysis of structurally related and previously reported acetylene-bridged PDI dimers. ${ }^{29}$ 


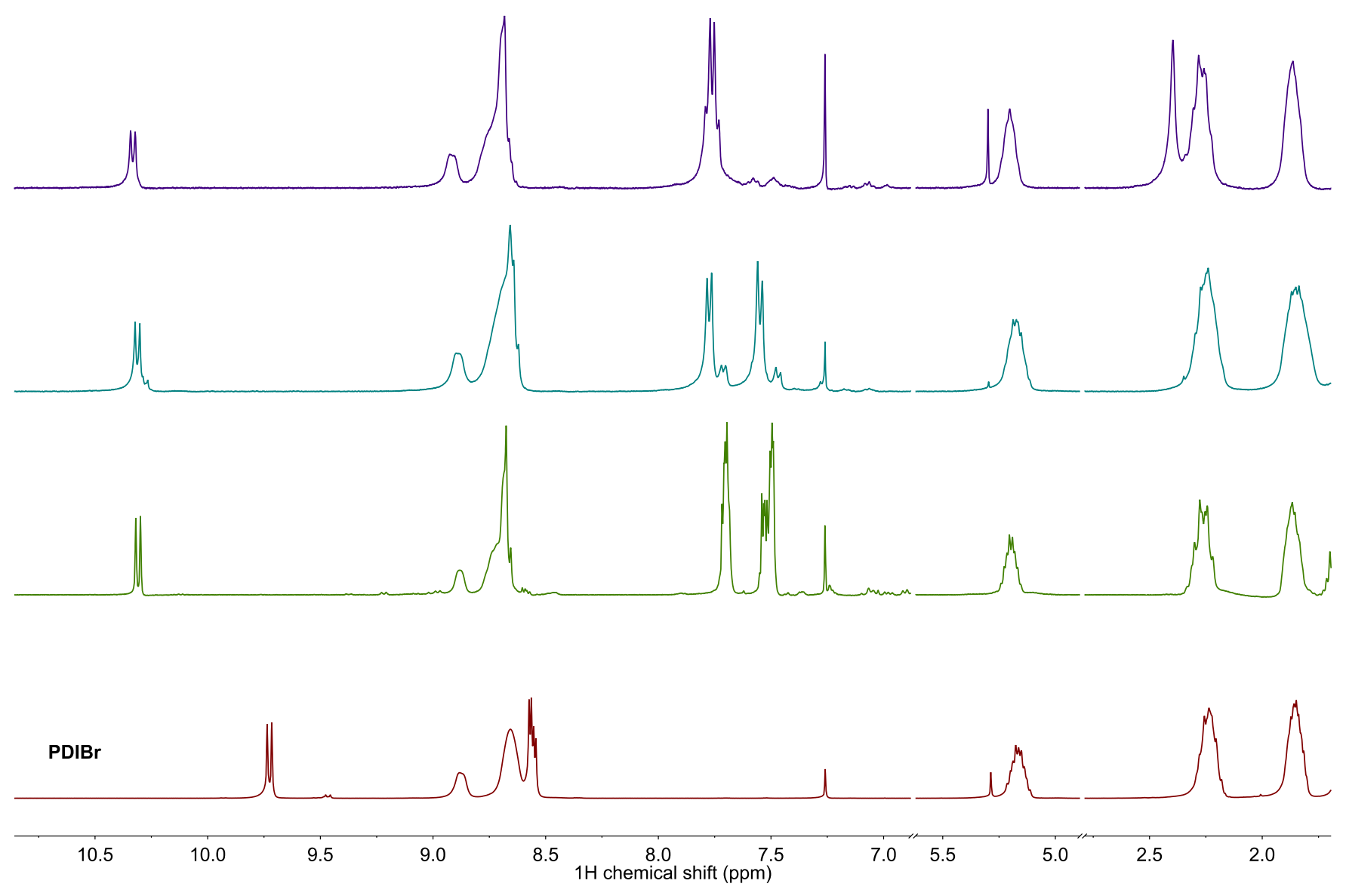

Figure 2. Comparison of ${ }^{1} \mathrm{H}$ NMR spectra (400 MHz; $\mathrm{CDCl}_{3} ; 295 \mathrm{~K}$ ) for PDI tetramers 1 and 2 , monomer 3 and PDIBr. For clarity, only the regions of interest are shown. All chemical shifts are referenced to the residual solvent peak $\left(\mathrm{CDCl}_{3}, \delta 7.26 \mathrm{ppm}\right)$. The representative chemical structures are shown in Figure 1 and Supplementary Data.

At room temperature (295 K), both PDI tetramers (1 and 2) and PDI monomer 3 displayed very similar ${ }^{1} \mathrm{H}$ NMR patterns with partial broadening of aromatic resonances: two well separated P12 and P2 protons and a broad chemical shift envelope comprising of 5 protons (P5-P8 and P11). This splitting pattern is quite characteristic and has been observed in other related PDI-based assemblies. Structurally, the positioning of acetylene group induces a strong deshielding effect on its spatially most proximate proton (P12) that appears as the most downfield shifted doublet ( $\delta 10.30 \mathrm{ppm})$. This effect is further evidenced upon the comparison of ${ }^{1} \mathrm{H}$ NMR spectra (Figure 2): the attachment of a phenylethynyl $(\mathrm{CCPh})$ arm to the 1-position of PDI causes the substantial downfield shift of P12 proton $(\sim 0.5 \mathrm{ppm})$ relative to $\mathrm{PDIBr}$, whereas all other remaining perylene-based protons experience relatively minor 
changes. Another accompanying effect is the downfield shift of the phenyl group resonances $(\sim 0.4 \mathrm{ppm})$ in PDI tetramers 1 and 2 as compared to their rigid core precursors; for instance, in 1 vs. M4 (Supplementary Data Figure S10). In addition to that, in PDI tetramer 2 the methylene protons of adamantane core $(\delta 2.39 \mathrm{ppm})$ also experience a deshielding effect and are downfield shifted by $\sim 0.65$ ppm relative to unsubstituted adamantane (Supplementary Data Figure S12). At room temperature (295 $\mathrm{K}), \mathrm{P} 12$ proton is a relatively well resolved doublet $(\delta 10.30 \mathrm{ppm}, J=8.2 \mathrm{~Hz})$ while $\mathrm{P} 2$ proton appears as a broad singlet (e.g., $\delta 8.96-8.87 \mathrm{ppm}$ for 2 ) stemming from the underlying chemical exchange and therefore can be used as a convenient probe to evaluate the kinetic parameters of this phenomenon (Figure 4 and Table 1).

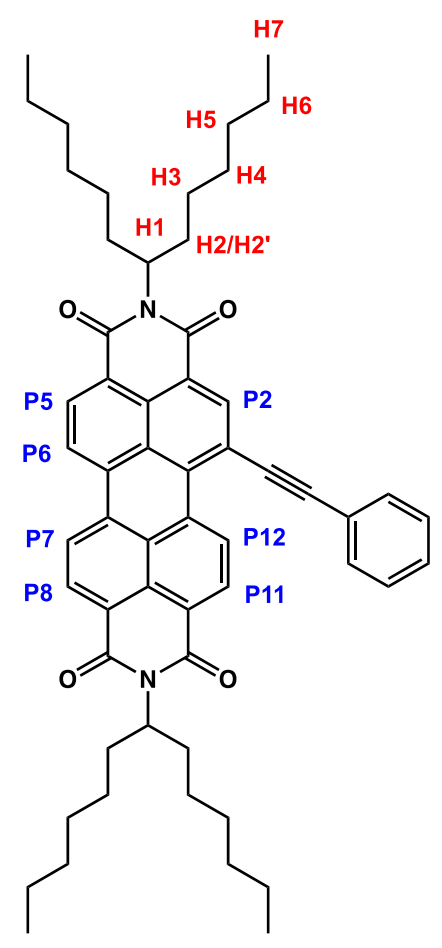

Figure 3. Atom-labeling scheme used for the chemical shift assignment in ${ }^{1} \mathrm{H}$ NMR spectra of PDI tetramers 1 and 2 , and monomer 3 . PDI monomer 3 is shown as a representative example.

The aliphatic 1-hexylheptyl group remains pseudo-symmetric even when the perylene core symmetry is perturbed upon the incorporation of $\mathrm{CCPh}$ arm in 1-position of its bay region. The $\mathrm{H} 1$ protons next to the imide nitrogen $(\delta 5.24-5.16 \mathrm{ppm})$ are characteristic of a wide variety of PDI molecules in general 
and can be conveniently used as integration reference points. Based on our previous studies of structurally relevant acetylene-bridged PDI dimers using multidimensional NMR spectroscopy (e.g., heteronuclear ${ }^{1} \mathrm{H}^{13}{ }^{13} \mathrm{C}$ HSQC experiments), protons $\mathrm{H} 2$ ( $\left.\delta 2.40-2.22 \mathrm{ppm}\right)$ and $\mathrm{H} 2$ ' ( $\left.\delta 1.92-1.82 \mathrm{ppm}\right)$ were found to exhibit a pronounced diastereotopic splitting accompanied by a substantial chemical shift difference $(\sim 0.4 \mathrm{ppm}){ }^{29}$

2.2.1. Perylene-centered conformational dynamics. In order to unravel the origin of spectral broadening in conventional ${ }^{1} \mathrm{H}$ NMR spectra (Figure 2) and higher than expected number of ${ }^{13} \mathrm{C}$ resonances (see Supplementary Data) observed at room temperature $(295 \mathrm{~K})$ in the synthesized monomeric and tetrameric PDIs (1, 2 and 3), we recorded the corresponding variable temperature (VT) ${ }^{1} \mathrm{H}$ NMR spectra $\left(\mathrm{CDCl}_{3}\right)$ in 253-328 K range (Figure 4 and Supplementary Data). As a representative example, the temperature-dependent behavior of PDI tetramer 2 will be discussed here.

As clearly evident from the temperature-induced changes in the aromatic regions (especially for protons P2 and P12), both PDI tetramers (1 and 2) and monomer 3 exist in a dynamic equilibrium arising primarily from the conformational dynamics of the perylene core (Figure 4 and Supplementary Data). ${ }^{29,58,59}$ On the other hand, the ${ }^{1} \mathrm{H}$ signals in the aliphatic regions $(\mathrm{H} 1-\mathrm{H} 7)$ were found to undergo very minor temperature-induced changes and, consequently, any possible rotational conformers could not be sufficiently resolved under the employed experimental conditions.

The $\mathrm{P} 2$ proton $(\delta 8.96-8.87)$ is structurally unique as it is the only uncoupled aromatic proton within the perylene core and therefore can best serve as a probe of the dynamic behavior of 2 . Based on the obtained VT NMR data, the dynamic behavior of all synthesized PDI molecules (1, 2 and 3) can be rationally described by a two-state conformational equilibrium model with nearly equal population distributions. ${ }^{60}$ At temperatures below RT (253-283 K), P2 proton appears as a set of 2 singlets of nearly equal intensity with a peak separation of $\sim 17.5 \mathrm{~Hz}$. This represents a textbook example of a dynamic spin system that exists in the slow exchange regime and two different conformers are clearly resolved. The coalescence point emerges near RT (295 K) and the P2 peak separation is no longer resolved, thus indicating a crossover into the fast exchange regime. Above this point $(>300 \mathrm{~K})$, the system shifts 
completely into the fast exchange mode and the PDI conformational dynamics becomes too fast to be resolved into two separate components. Consequently, the P2 proton gradually becomes a well-defined ensemble-average singlet in complete accordance with its structural placement.

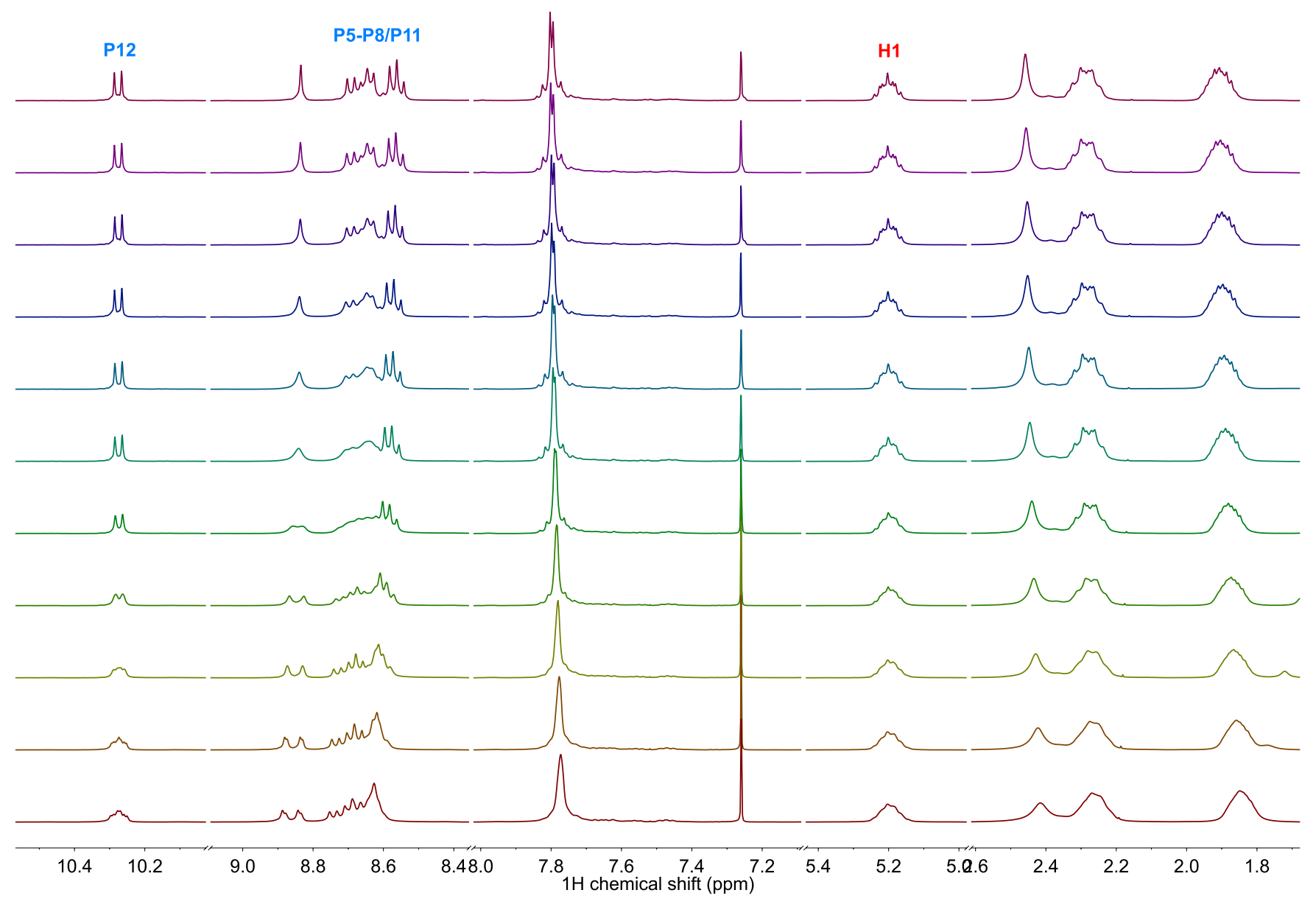

Figure 4. Temperature-dependent ${ }^{1} \mathrm{H}$ NMR spectra of PDI tetramer $2\left(400 \mathrm{MHz}, \mathrm{CDCl}_{3}\right)$ measured in 253-328 K range. For clarity, only the regions of interest are displayed.

The residual exchange-induced line broadening of $\mathrm{P} 2$ proton can be conveniently used to estimate the rate constants $\left(k_{\mathrm{ex}}\right)$ and the activation barrier $\Delta G^{\ddagger}$ ) for the observed conformational exchange (Figure 5). The estimated activation energy for both PDI tetramers (1 and 2) and monomer 3 was found to be within 15-17 kcal/mol (Table 1) that falls right in the ballpark of literature reported values for structurally relevant molecules. ${ }^{29,47,59,61-63}$ Furthermore, the origin of the observed conformational exchange has been often attributed to the induced out-of-plane twist of the perylene core upon the 
chemical modification of its bay region, with dihedral angles ranging from $13^{0}$ to $37^{0}$ depending on the number and nature of the substituent groups. ${ }^{29,64-66}$ In other words, the origin of the observed conformational dynamics in PDI tetramers 1 and 2 , and monomer 3 comes from the local fluctuations in the twist (dihedral) angle of the perylene core. It is important to note here that the conformational dynamics of PDI tetramers is only observable by solution NMR spectroscopy. In addition, the concentration-dependent NMR studies (Supplementary Data Figures S15 and S16) showed no evidence of any aggregation-induced effects (which, if existed, would be typically accompanied by the substantial chemical shift changes) in a measured concentration range of 0.1-2 $\mathrm{mM}$ and thus confirmed that the observed broadening of ${ }^{1} \mathrm{H}$ NMR signals in PDI tetramers 1 and 2 stems solely from the conformational dynamics of twisted perylene core(s) within their corresponding phenylethynyl-perylenediimide ( $\mathrm{PDICCPh}$ ) subunits. Noteworthy, chlorinated solvents (such as $\mathrm{CH}_{2} \mathrm{Cl}_{2}$ or $\mathrm{CHCl}_{3}$ ) are well known to be "good" solvents for PDI-based systems as they usually tend to prevent these chromophores from aggregating. On a separate note, it is quite interesting that the observed perylene-centered conformational exchange phenomenon seems to be amplified when PDI-based chromophoric assemblies incorporate long 1-hexylheptyl solubilizing groups whereas no spectral broadening (at RT) was observed with shorter (e.g., 1-ethylpropyl) substituents (data not shown). However, the exact origin of this effect is not yet well understood. 


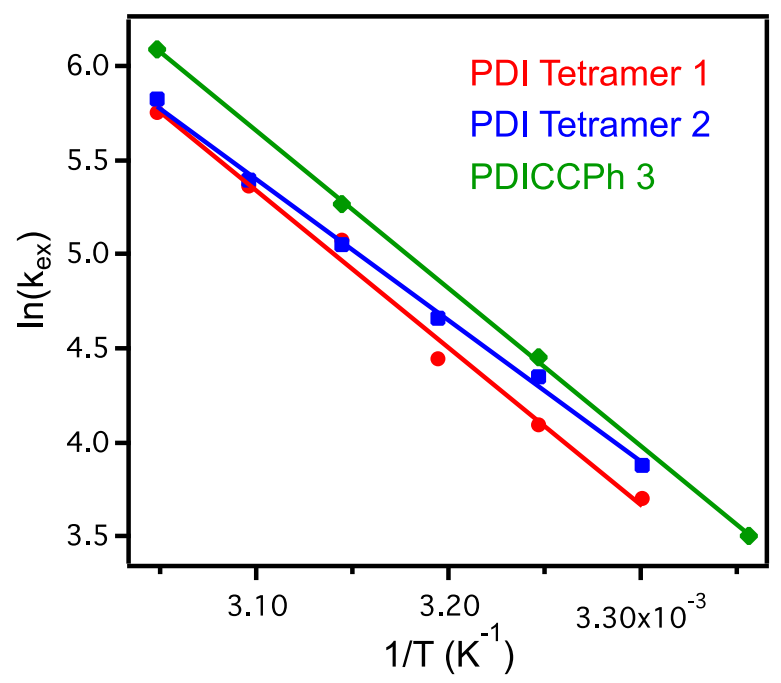

Figure 5. Arrhenius analysis of conformational exchange (dynamic NMR) in PDI tetramers 1 and 2 , and monomer 3 .

Table 1. Calculated conformational exchange kinetic parameters for PDI tetramers 1 and 2 , and monomer 3.

\begin{tabular}{|c|c|c|c|}
\hline PDI compound & Temperature $(\mathrm{K})$ & $k_{\mathrm{ex}}\left(\mathrm{s}^{-1}\right)$ & $\Delta G^{\ddagger}(\mathrm{kcal} / \mathrm{mol})$ \\
\hline \multirow{3}{*}{1} & $303 \pm 0.1$ & $40.65 \pm 2.8$ & \\
& $308 \pm 0.1$ & $59.75 \pm 3.5$ & $16.6 \pm 0.2$ \\
& $313 \pm 0.1$ & $84.92 \pm 4.1$ & \\
& $318 \pm 0.1$ & $159.79 \pm 4.8$ & \\
\hline \multirow{2}{*}{2} & $323 \pm 0.1$ & $213.99 \pm 5.7$ & \\
& $328 \pm 0.1$ & $315.44 \pm 7.3$ & \\
& $303 \pm 0.1$ & $78.21 \pm 2.3$ & \\
& $308 \pm 0.1$ & $105.73 \pm 3.4$ & \\
& $313 \pm 0.1$ & $155.73 \pm 4.8$ & \\
& $318 \pm 0.1$ & $220.37 \pm 6.1$ & \\
& $323 \pm 0.1$ & $338.53 \pm 7.6$ & \\
& $328 \pm 0.1$ & $33.26 \pm 2.2$ & \\
& $298 \pm 0.1$ & $85.76 \pm 3.8$ & \\
& $308 \pm 0.1$ & $194.44 \pm 4.9$ & \\
& $318 \pm 0.1$ & $440.61 \pm 6.4$ & \\
\hline
\end{tabular}

2.3. Photophysical properties. The photophysical properties of target PDI molecules (1-3) have been measured in dichloromethane (DCM). In general, PDI tetramers 1 and 2 possess exceptional (photo)chemical stability and their photophysical properties are characteristic of a wide variety of PDIbased molecular systems. ${ }^{1,2}$ The steady-state absorption spectra are presented in Figure 6 (left). The absorption bands in the near-UV region (300-400 nm) are assigned to the $\pi-\pi^{*}$ transitions of the 
phenylethynyl $(\mathrm{CCPh})$ arm whereas PDI-centered $\pi-\pi^{*}$ transitions span the visible portion of the spectrum (400-600 nm). Compared to PDIBr (see Supplementary Data Figure S17), the attachment of the phenylethynyl $(\mathrm{CCPh})$ group to the 1-position of PDI bay region results in a bathochromic shift of PDI-centered $\pi-\pi^{*}$ transitions in the visible and enhancement of the light-harvesting properties of 3 in near-UV region. However, the delocalization of electron density over the entire PDICCPh moiety inadvertently leads to a decrease of the oscillator strength of the PDI-centered $\pi-\pi^{*}$ transitions, as compared to the unsubstituted PDI (used here as a reference): $53,000 \mathrm{M}^{-1} \mathrm{~cm}^{-1}$ for $3 \mathrm{vs} .88,000 \mathrm{M}^{-1} \mathrm{~cm}^{-1}$ for PDI (Table 2). ${ }^{1,50}$ Compared to monomer 3, the absorption spectra of PDI tetramers 1 and 2 are only marginally red-shifted $(\sim 2 \mathrm{~nm})$ and somewhat broadened on the lower energy side (see Supplementary Data Figure S18). However, integration of the data presented in Figure 6 (left) between 300-600 nm indicates a non-linear enhancement (larger than a factor of 4) of electronic transitions in PDI tetramers 1 and 2, as compared to monomer 3 (Table 2). Both lower energy, PDI-centered and higher energy, phenylethynyl-centered $\pi-\pi^{*}$ transitions contribute to the enhancement of light-harvesting properties in the newly synthesized PDI tetramers. Based on our recent photophysical studies of acetylene-bridged PDI dimers using coherent two-dimensional electronic spectroscopy, we hypothesize that the observed absorption enhancement effect may stem from intramolecular excitonic coupling between the adjacent PDI subunits within the tetramer framework and thus will be the subject of in-depth photophysical investigation in a separate future contribution. ${ }^{19}$ Interestingly, this enhancement is the largest in PDI tetramer 1 (Table 2) and may indicate a stronger degree of intramolecular interactions between neighboring PDI subunits when they are attached to a smaller, methane-centered rigid core. 

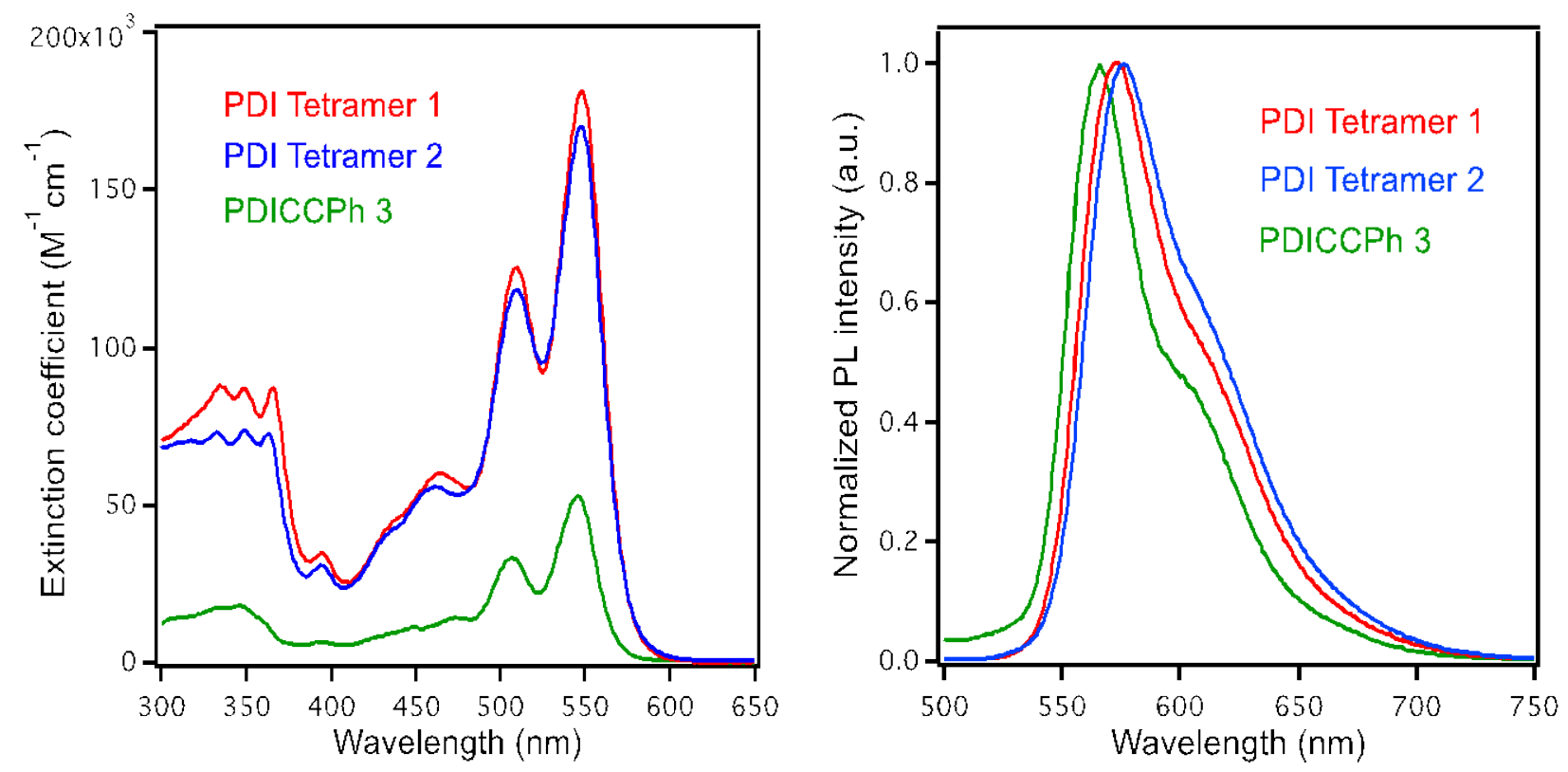

Figure 6. Steady-state absorption (left) and uncorrected emission (right, $\lambda_{\mathrm{ex}}=480 \mathrm{~nm}$ ) spectra of PDI tetramers 1 (red) and 2 (blue), and monomer 3 (green) measured in dichloromethane.

The photoluminescence of PDI tetramers 1 and 2, and monomer 3 originates from the singlet excited state and is characteristic of other related PDI-based chromophores (Figure 6, right). ${ }^{41}$ The emission spectra of both PDI tetramers (1 and 2$)$ are nearly superimposable and their emission maxima $\left(\lambda_{\max } \sim\right.$ $574 \mathrm{~nm})$ are slightly red-shifted $(\sim 8 \mathrm{~nm})$ as compared to monomer $3\left(\lambda_{\max }=566 \mathrm{~nm}\right)$. The apparent loss of the vibronic structure in the emission spectra is likely due to the delocalization of electron density over the entire $\mathrm{PDICCPh}$ subunit. As compared to monomer 3, the tetrahedral assembly of four PDICCPh subunits into PDI tetramers 1 and 2 causes an enhancement of non-radiative decay pathways as evidenced by the substantial drop $(>10 \%)$ of their corresponding fluorescence quantum yields $\left(\Phi_{\mathrm{FL}}\right.$, Table 2). In addition, time-resolved photoluminescence measurements were performed to further characterize the photophysical behavior of PDI tetramers 1 and 2 (Figure 7). Under optically dilute conditions in dichloromethane, PDI tetramers 1 and 2, and monomer 3 were found to exhibit monoexponential fluorescence decays, with each PDI tetramer possessing slightly longer excited state lifetime than monomer 3: 6.9 ns vs. 6.1 ns (Supplementary Data Figure S19 and Table 2). To summarize, the photophysical properties of these newly synthesized PDI tetramers 1 and 2 appear to be 
consistent with other related PDI-based assemblies; however, as we noted before, a more detailed photophysical investigation of these PDI tetramers will be reported in a separate contribution.

Table 2. Photophysical properties of PDI tetramers 1 and 2 , and monomer 3 measured in dichloromethane.

\begin{tabular}{|l|c|c|c|}
\hline \multirow{2}{*}{} & \multicolumn{2}{|c|}{ PDI compound } \\
\cline { 2 - 4 } & 1 & 2 & 3 \\
\hline Extinction coefficient at $\lambda_{\max }\left(\mathrm{M}^{-1} \mathrm{~cm}^{-1}\right)$ & $181,000 \pm 2,000$ & $169,000 \pm 2,000$ & $53,000 \pm 3,000$ \\
\hline Relative absorption integrated area (a.u.) & $4.48 \pm 0.01$ & $4.16 \pm 0.01$ & $1.0 \pm 0.06$ \\
\hline Fluorescence lifetime $\left(\tau_{\mathrm{FL}}, \mathrm{ns}\right)$ & $6.9 \pm 0.1$ & $6.9 \pm 0.1$ & $6.1 \pm 0.1$ \\
\hline Fluorescence quantum yield $\left(\Phi_{\mathrm{FL}}\right)$ & $0.59 \pm 0.03$ & $0.58 \pm 0.03$ & $0.71 \pm 0.03$ \\
\hline $\mathrm{k}_{\mathrm{r}}\left(\mathrm{s}^{-1}\right)$ & $0.82 \times 10^{8}$ & $0.84 \times 10^{8}$ & $1.16 \times 10^{8}$ \\
\hline $\mathrm{k}_{\mathrm{nr}}\left(\mathrm{s}^{-1}\right)$ & $0.57 \times 10^{8}$ & $0.61 \times 10^{8}$ & $0.48 \times 10^{8}$ \\
\hline $\mathrm{k}_{\mathrm{r}} / \mathrm{k}_{\mathrm{nr}}$ & 1.44 & 1.38 & 2.42 \\
\hline
\end{tabular}

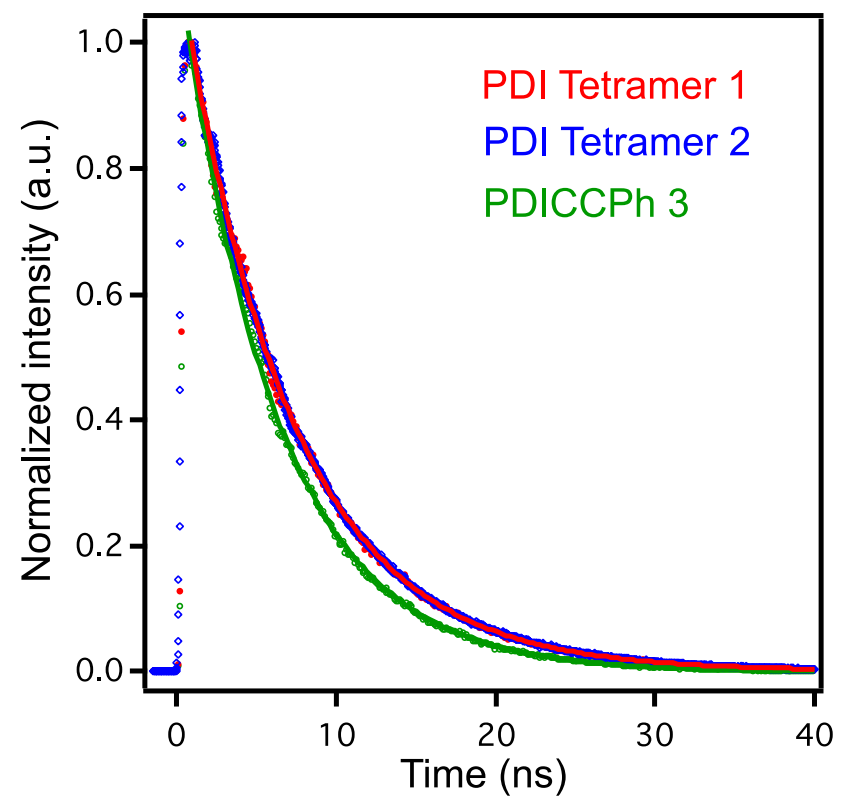

Figure 7. Fluorescence intensity decays of PDI tetramers 1 (red) and 2 (blue), and monomer 3 (green) measured in optically dilute dichloromethane solutions $\left(\lambda_{\mathrm{ex}}=480 \mathrm{~nm} ; \lambda_{\mathrm{em}}=570 \mathrm{~nm}\right)$.

\section{Conclusions}

We have synthesized and thoroughly structurally characterized two new representative tetrahedral rigid core "antenna" tetramers bearing phenylethynyl-perylenediimide (PDICCPh) subunits. The 
developed synthetic methodology is expected to be a valuable addition to the synthetic toolbox geared towards the design of novel complex multichromophoric systems. Dynamic NMR experiments revealed the existence of perylene-centered dynamic conformational exchange $\left(\Delta G^{ \pm}=15-17 \mathrm{kcal} / \mathrm{mol}\right)$, the primary cause of substantial spectral broadening of the aromatic resonances in conventionally acquired (RT) ${ }^{1} \mathrm{H}$ NMR spectra. Interestingly, the observed perylene-centered conformational exchange phenomenon appears to be a common theme for a variety of related PDI-based chromophoric assemblies that incorporate long 1-hexylheptyl solubilizing groups. Preliminary photophysical characterization of PDI tetramers 1 and 2 pointed towards an underlying intramolecular interaction (likely excitonic coupling) between the neighboring PDICCPh subunits and will be the subject of our future research efforts.

\section{Experimental}

4.1. General. Most synthetic manipulations were carried out under inert atmosphere (nitrogen) using standard Schlenk techniques unless otherwise noted. All solvents (ACS reagent grade) and reagents were purchased from commercial sources and used as received. When necessary, anhydrous solvents were obtained from a standard solvent purification system equipped with activated alumina columns. To ensure the reproducibility of reaction procedures, all reaction sequences were repeated at least two times.

4.2. Characterization and instrumentation. The structures of synthesized phenylethynylperylenediimide tetramers 1 and 2 along with monomer 3 were confirmed by high-resolution ${ }^{1} \mathrm{H}$ and ${ }^{13} \mathrm{C}$ NMR spectroscopy, MALDI-TOF mass spectrometry (MALDI-MS) and elemental analysis. Mass spectra were acquired using a standard MALDI-TOF spectrometer (MSU Mass Spectrometry Facility). Elemental analysis was performed by Atlantic Microlab, Inc. ${ }^{1} \mathrm{H}(400 \mathrm{MHz})$ and ${ }^{13} \mathrm{C}(100 \mathrm{MHz}$ and 176 MHz) were recorded at $295 \mathrm{~K}$ on $400 \mathrm{MHz}$ Varian Inova spectrometer and $700 \mathrm{MHz}$ Bruker Avance 1 spectrometer. Variable-temperature (VT) ${ }^{1} \mathrm{H}$ NMR (400 MHz) experiments were carried out in 253-328 $\mathrm{K}$ range with sample temperature equilibration time of 10 minutes. NMR spectral analysis was 
performed using MestReNova 9.0.1 software. All chemical shifts were referenced to the residual solvent peak $\left(\mathrm{CDCl}_{3}, \delta 7.26 \mathrm{ppm}\right.$ for ${ }^{1} \mathrm{H}$ and $77.23 \mathrm{ppm}$ for $\left.{ }^{13} \mathrm{C}\right)$ and splitting patterns were assigned as $\mathrm{s}$ (singlet), $\mathrm{d}$ (doublet), and $\mathrm{m}$ (multiplet). The conformational exchange rate constants $\left(k_{\mathrm{ex}}\right)$ were calculated from the lineshape broadening deconvolution of an uncoupled aromatic proton (P2) in 298 $328 \mathrm{~K}$ temperature range (fast exchange regime) assuming a two-state, equal population exchange model and using the following equation:

$$
k_{e x}=\frac{\pi\left(v_{A}-v_{B}\right)^{2}}{2\left(L W_{A B}-L W_{R E F}\right)}
$$

where: $\left(v_{A}-v_{B}\right)$ is the exchangeable uncoupled proton peak (P2) separation (in $\mathrm{Hz}$ ) measured at $253 \mathrm{~K}$ (slow exchange regime); $L W_{A B}$ is a linewidth (FWHM, in $\mathrm{Hz}$ ) of the uncoupled exchangeable proton peak (P2) at a given temperature; $L W_{R E F}$ is the linewidth (FWHM, in $\mathrm{Hz}$ ) of a reference nonexchangeable proton peak (residual solvent peak, $\mathrm{CDCl}_{3}$ ) at a given temperature. The activation energy $\left(\Delta G^{\ddagger}\right)$ for the conformational exchange was estimated from the Arrhenius analysis $\left(\ln \left(k_{\mathrm{ex}}\right)\right.$ vs. $\left.1 / T\right)$.

The steady-state absorption spectra were measured in optically dilute dichloromethane solutions on Shimadzu UV-3600 UV-Vis-NIR spectrophotometer. The extinction coefficients were calculated as an average of two independent runs. The uncorrected steady-state emission spectra were measured in optically dilute, air-saturated dichloromethane solutions on Edinburgh Instruments FLS980 fluorescence spectrometer. The fluorescence quantum yields $\left(\Phi_{F L}\right)$ were measured in dichloromethane using N,N'bis(1-hexylheptyl)-perylene-3,4:9,10-tetracarboxylic diimide (PDI) as a reference. The excited state lifetimes $\left(\tau_{F L}\right)$ were measured using the gated second-harmonic ( $4 \mathrm{MHz}, \sim 1 \mathrm{~nJ} /$ pulse) output of a tunable ultrafast Ti-Sapphire oscillator (Coherent, $\sim 120 \mathrm{fs}$ ) as an excitation source and a time-correlated single photon counting (TCSPC) detection system (LifeSpec II, Edinburgh Instruments, 100 ps IRF), and estimated from the first-order decay fit of single wavelength emission transients using Igor Pro 6.36 software. The radiative $\left(k_{r}\right)$ and non-radiative $\left(k_{n r}\right)$ excited state decay rate constants were calculated as follows: 


$$
k_{r}=\frac{\Phi_{F L}}{\tau_{F L}} \quad k_{n r}=\frac{1}{\tau_{F L}}-k_{r}
$$

4.3. Synthesis. Tetrahedral methane- and adamantane-centered rigid cores were synthesized via multistep procedures using modified literature protocols. All intermediates and target products were isolated using standard workup and purification techniques. All synthesized rigid core intermediates and PDI synthons produced satisfactory analytical characterization data that were found to be consistent with previously reported values.

\subsubsection{Tetrakis-[1-(4-ethynyl-phenyl)-N,N'-bis(1-hexylheptyl)-perylene-3,4:9,10-tetracarboxylic}

diimide]-methane (1). $50 \mathrm{mg}(0.12 \mathrm{mmol})$ of tetrakis(4-ethynylphenyl)methane (M4), $440 \mathrm{mg}(0.53$ mmol) of 1-bromoperylenediimide (PDIBr), $18 \mathrm{mg}(0.1 \mathrm{mmol})$ of CuI and $100 \mathrm{mg}(0.1 \mathrm{mmol})$ of $\operatorname{Pd}\left(\mathrm{PPh}_{3}\right)_{4}$ were placed under nitrogen in a $100 \mathrm{~mL}$ Schlenk flask equipped with a reflux condenser, followed by the addition of $10 \mathrm{~mL}$ of $\mathrm{N}_{2}$-degassed diisopropylamine (anh.) and $20 \mathrm{~mL}$ of dichloromethane (anh.). The reaction mixture was additionally degassed with $\mathrm{N}_{2}$ for 20 min. and then heated to $65{ }^{0} \mathrm{C}$ for 48 hours (under nitrogen). The reaction progress was monitored by steady-state absorption spectroscopy. After completion, the reaction mixture was cooled to RT and then quenched with $10 \% \mathrm{HCl}$. Afterwards, the product was extracted with dichloromethane, combined organic fractions dried over $\mathrm{Na}_{2} \mathrm{SO}_{4}$ (anh.), filtered and concentrated under vacuum. The obtained solid was recrystallized at least twice from dichloromethane/methanol (1:3) via liquid diffusion to afford analytically pure dark red solid in $64 \%$ yield $(263 \mathrm{mg})$. MALDI-MS: $m / z=3429.7\left([\mathrm{M}+\mathrm{H}]^{+}\right)$. Anal. Calcd for $\mathrm{C}_{233} \mathrm{H}_{260} \mathrm{~N}_{8} \mathrm{O}_{16}: \mathrm{C}, 81.62 ; \mathrm{H}, 7.64 ; \mathrm{N}, 3.27$. Found: $\mathrm{C}_{233} \mathrm{H}_{260} \mathrm{~N}_{8} \mathrm{O}_{16}: \mathrm{C}, 81.53 ; \mathrm{H}, 7.51 ; \mathrm{N}, 3.26$. ${ }^{1} \mathrm{H}$ NMR (400 MHz, CDCl $\left.3,295 \mathrm{~K}\right): 10.31(4 \mathrm{H}, \mathrm{d}, J=8.3 \mathrm{~Hz}) ; 8.92-8.86$ (4H, broad s); 8.77-8.61 (20H, m); 7.78-7.70 (4H, dd, $J=8.2 \mathrm{~Hz}) ; 7.56-7.48(4 \mathrm{H}, \mathrm{dd}, J=8.2 \mathrm{~Hz}) ; 5.22-5.11(8 \mathrm{H}, \mathrm{m}) ; 2.30-2.15(16 \mathrm{H}$, m); 1.93-1.75 (16H, m); 1.42-1.12 (128H, m); 0.87-0.73 (48H, m). ${ }^{13} \mathrm{C}$ NMR (176 MHz, $\mathrm{CDCl}_{3}, 295$ K): $164.9 ; 164.7 ; 164.6 ; 164.3 ; 163.8 ; 163.7 ; 163.6 ; 163.2 ; 147.2 ; 138.9 ; 138.2 ; 134.5 ; 134.1 ; 132.0$; $131.9 ; 131.6 ; 131.0 ; 130.8 ; 129.3 ; 128.7 ; 127.4 ; 127.2 ; 126.9 ; 124.3 ; 124.1 ; 123.8 ; 123.7 ; 123.5 ; 123.3$; 
$123.1 ; 123.0 ; 122.3 ; 121.1 ; 120.9 ; 120.3 ; 99.3 ; 92.0 ; 65.7 ; 55.1 ; 54.9 ; 32.5 ; 32.0 ; 31.9 ; 29.4 ; 27.1 ; 27.0$;

$22.8 ; 22.7 ; 14.3 ; 14.2$.

\subsubsection{Tetrakis-1,3,5,7-[1-(4-ethynyl-phenyl)-N,N'-bis(1-hexylheptyl)-perylene-3,4:9,10-}

tetracarboxylic diimide]-adamantane (2). $50 \quad \mathrm{mg} \quad(0.093 \quad \mathrm{mmol})$ of 1,3,5,7-tetrakis(4ethynylphenyl)adamantane (A5), $375 \mathrm{mg}$ (0.45 mmol) of 1-bromoperylenediimide (PDIBr), $18 \mathrm{mg}(0.1$ mmol) of $\mathrm{CuI}$ and $100 \mathrm{mg}(0.1 \mathrm{mmol})$ of $\mathrm{Pd}\left(\mathrm{PPh}_{3}\right)_{4}$ were placed under nitrogen in a $100 \mathrm{~mL}$ Schlenk flask equipped with a reflux condenser, followed by the addition of $10 \mathrm{~mL}$ of $\mathrm{N}_{2}$-degassed diisopropylamine (anh.) and $20 \mathrm{~mL}$ of dichloromethane (anh.). The reaction mixture was additionally degassed with $\mathrm{N}_{2}$ for $20 \mathrm{~min}$. and then heated to $65{ }^{\circ} \mathrm{C}$ for 48 hours (under nitrogen). The workup and purification protocols were identical to 1. After recrystallization, PDI tetramer 2 was isolated as analytically pure dark red solid in $75 \%$ yield $(247 \mathrm{mg})$. We also note that any residual (emissive) impurities in PDI tetramers 1 and $\mathbf{2}$ can be removed by careful repetitive precipitation of concentrated DCM solution(s) into excess hexane. MALDI-MS: $m / z=3549.9\left([\mathrm{M}+\mathrm{H}]^{+}\right)$. Anal. Calcd for $\mathrm{C}_{242} \mathrm{H}_{272} \mathrm{~N}_{8} \mathrm{O}_{16} \cdot \mathrm{CH}_{2} \mathrm{Cl}_{2}: \mathrm{C}, 80.32 ; \mathrm{H}, 7.60 ; \mathrm{N}, 3.08$. Found: C, 80.08; H, 7.45; N, 3.06. ${ }^{1} \mathrm{H}$ NMR (400 $\left.\mathrm{MHz}, \mathrm{CDCl}_{3}, 295 \mathrm{~K}\right): 10.33(4 \mathrm{H}, \mathrm{d}, J=8.2 \mathrm{~Hz}) ; 8.96-8.87$ (4H, broad s); 8.81-8.63 (20H, m); 7.83-7.70 $(16 \mathrm{H}, \mathrm{m}) ; 5.24-5.16(8 \mathrm{H}, \mathrm{m}) ; 2.40-2.22(28 \mathrm{H}, \mathrm{m}) ; 1.92-1.82(16 \mathrm{H}, \mathrm{m}) ; 1.40-1.15$ (128H, m); 0.84-0.79 (48H, m). ${ }^{13} \mathrm{C}$ NMR (176 MHz, $\left.\mathrm{CDCl}_{3}, 295 \mathrm{~K}\right): 164.9 ; 164.7 ; 164.3 ; 163.8 ; 163.6 ; 163.2 ; 150.8 ; 139.0$; $138.3 ; 134.5 ; 134.1 ; 132.4 ; 131.7 ; 131.6 ; 130.9 ; 130.8 ; 129.3 ; 128.6 ; 127.4 ; 127.1 ; 126.8 ; 126.0 ; 124.2 ;$ $124.0 ; 123.8 ; 123.6 ; 123.5 ; 123.3 ; 123.2 ; 123.1 ; 122.9 ; 122.2 ; 120.6 ; 120.4 ; 100.0 ; 91.4 ; 55.1 ; 54.9$; $47.1 ; 40.0 ; 32.6 ; 32.0 ; 29.5 ; 27.2 ; 22.8 ; 22.7 ; 14.3$.

Acknowledgments. This work was financially supported by the Air Force Office of Scientific Research (FA9550-13-1-0106). We thank Dr. Valentina Prusakova (BGSU) for valuable input at the preliminary stages of this project, and Dr. Xiaoyan Sun (NCSU) for the assistance with dynamic NMR experiments.

Supplementary Data. Representative synthetic scheme and synthetic protocol for a model monomer 3 (PDICCPh); ${ }^{1} \mathrm{H}$ and ${ }^{13} \mathrm{C}$ NMR spectra of PDI tetramers 1 and 2, and monomer 3; MALDI-TOF spectra 
of 1 and $2 ;{ }^{1} \mathrm{H}$ NMR spectra of rigid core intermediates in the synthetic sequences towards M4 and A5;

temperature-dependent ${ }^{1} \mathrm{H}$ NMR spectra of 1 and 3; concentration-dependent ${ }^{1} \mathrm{H}$ NMR spectra of 1 and

2; additional steady-state and time-resolved photophysical data.

\section{References}

(1) Langhals, H. Helv. Chim. Acta 2005, 88, 1309.

(2) Wurthner, F. Chem. Commun. 2004, 1564.

(3) Castellano, F. N. Dalton Trans. 2012, 41, 8493.

(4) Zhan, X. W.; Facchetti, A.; Barlow, S.; Marks, T. J.; Ratner, M. A.; Wasielewski, M. R.; Marder, S. R. Adv. Mater. 2011, 23, 268.

9068.

(5) Weil, T.; Vosch, T.; Hofkens, J.; Peneva, K.; Mullen, K. Angew. Chem. Int. Ed. 2010, 49,

(6) Würthner, F.; Saha-Möller, C. R.; Fimmel, B.; Ogi, S.; Leowanawat, P.; Schmidt, D. Chem. Rev. 2015. DOI: 10.1021/acs.chemrev.5b00188

(7) Hartnett, P. E.; Timalsina, A.; Matte, H. S. S. R.; Zhou, N.; Guo, X.; Zhao, W.; Facchetti, A.; Chang, R. P. H.; Hersam, M. C.; Wasielewski, M. R.; Marks, T. J. J. Am. Chem. Soc. 2014, 136, 16345.

(8) Wilson, T. M.; Tauber, M. J.; Wasielewski, M. R. J. Am. Chem. Soc. 2009, 131, 8952.

(9) Li, C.; Wonneberger, H. Adv. Mater. 2012, 24, 613.

(10) Deng, F.; Sommer, J. R.; Myahkostupov, M.; Schanze, K. S.; Castellano, F. N. Chem. Commun. 2013, 49, 7406.

(11) Yang, S. K.; Shi, X. H.; Park, S.; Doganay, S.; Ha, T.; Zimmerman, S. C. J. Am. Chem. Soc. 2011, 133, 9964.

(12) Singh-Rachford, T. N.; Nayak, A.; Muro-Small, M. L.; Goeb, S.; Therien, M. J.; Castellano, F. N. J. Am. Chem. Soc. 2010, 132, 14203.

(13) Takada, T.; Ido, M.; Ashida, A.; Nakamura, M.; Fujitsuka, M.; Kawai, K.; Majima, T.; Yamana, K. Chem.-Eur. J. 2015, 21, 6846.

(14) Schmidt, D.; Bialas, D.; Würthner, F. Angew. Chem. 2015, 127, 3682.

(15) Schulze, M.; Steffen, A.; Würthner, F. Angew. Chem. Int. Ed. 2015, 54, 1570.

(16) Rachford, A. A.; Goeb, S.; Castellano, F. N. J. Am. Chem. Soc. 2008, 130, 2766.

(17) Brown, K. E.; Veldkamp, B. S.; Co, D. T.; Wasielewski, M. R. J. Phys. Chem. Lett. 2012, 3, 2362.

(18) Li, X. Y.; Sinks, L. E.; Rybtchinski, B.; Wasielewski, M. R. J. Am. Chem. Soc. 2004, $126,10810$.

(19) Jumper, C. C.; Anna, J. M.; Stradomska, A.; Schins, J.; Myahkostupov, M.; Prusakova, V.; Oblinsky, D. G.; Castellano, F. N.; Knoester, J.; Scholes, G. D. Chem. Phys. Lett. 2014, 599, 23.

(20) Jimenez, A. J.; Grimm, B.; Gunderson, V. L.; Vagnini, M. T.; Calderon, S. K.; Rodriguez-Morgade, M. S.; Wasielewski, M. R.; Guldi, D. M.; Torres, T. Chem.-Eur. J. 2011, $17,5024$.

(21) Conron, S. M. M.; Shoer, L. E.; Smeigh, A. L.; Ricks, A. B.; Wasielewski, M. R. J. Phys. Chem. B2013, 117, 2195.

(22) Lindquist, R. J.; Lefler, K. M.; Brown, K. E.; Dyar, S. M.; Margulies, E. A.; Young, R. M.; Wasielewski, M. R. J. Am. Chem. Soc. 2014, 136, 14912.

(23) Supur, M.; Fukuzumi, S. J. Phys. Chem. C 2012, 116, 23274.

(24) Danilov, E. O.; Rachford, A. A.; Goeb, S.; Castellano, F. N. J. Phys. Chem. A2009, 113, 5763. 
(25) Yoo, H.; Furumaki, S.; Yang, J.; Lee, J. E.; Chung, H.; Oba, T.; Kobayashi, H.; Rybtchinski, B.; Wilson, T. M.; Wasielewski, M. R.; Vacha, M.; Kim, D. J. Phys. Chem. B 2012, 116, 12878.

(26) Singh-Rachford, T. N.; Castellano, F. N. Coord. Chem. Rev. 2010, 254, 2560.

(27) Fron, E.; Deres, A.; Rocha, S.; Zhou, G.; Mullen, K.; De Schryver, F. C.; Sliwa, M.; Ujii, H.; Hofkens, J.; Vosch, T. J. Phys. Chem. B2010, 114, 1277.

(28) Gomez, R.; Seoane, C.; Segura, J. L. J. Org. Chem. 2010, 75, 5099.

(29) Myahkostupov, M.; Prusakova, V.; Oblinsky, D. G.; Scholes, G. D.; Castellano, F. N. J. Org. Chem. 2013, 78, 8634.

(30) Sun, D.; Meng, D.; Cai, Y.; Fan, B.; Li, Y.; Jiang, W.; Huo, L.; Sun, Y.; Wang, Z. J. Am. Chem. Soc. 2015. DOI: 10.1021/jacs.5b06414

(31) Sonar, P.; Fong Lim, J. P.; Chan, K. L. Energy Environ. Sci. 2011, 4, 1558.

(32) Lin, Y.; Zhan, X. Mater. Horiz. 2014, 1, 470.

(33) Thyagarajan, S.; Galoppini, E.; Persson, P.; Giaimuccio, J. M.; Meyer, G. J. Langmuir 2009, 25, 9219.

(34) Lamberto, M.; Pagba, C.; Piotrowiak, P.; Galoppini, E. Tetrahedron Lett. 2005, 46, 4895.

(35) Galoppini, E. Coord. Chem. Rev. 2004, 248, 1283.

(36) Wei, Q.; Galoppini, E. Tetrahedron 2004, 60, 8497.

(37) Piotrowiak, P.; Galoppini, E.; Wei, Q.; Meyer, G. J.; Wiewiór, P. J. Am. Chem. Soc. 2003, 125, 5278.

(38) Shao, C. Z.; Grune, M.; Stolte, M.; Wurthner, F. Chem.-Eur. J. 2012, 18, 13665.

(39) Gorl, D.; Zhang, X.; Wurthner, F. Angew. Chem. Int. Ed. 2012, 51, 6328.

(40) Brown, K. E.; Salamant, W. A.; Shoer, L. E.; Young, R. M.; Wasielewski, M. R. J. Phys. Chem. Lett. 2014, 5, 2588.

16941.

(41) Ramanan, C.; Kim, C. H.; Marks, T. J.; Wasielewski, M. R. .J. Phys. Chem. C2014, 118,

(42) Echue, G.; Lloyd-Jones, G. C.; Faul, C. F. J. Chem.-Eur. J. 2015, 21, 5118.

(43) Chen, W.; Yang, X.; Long, G.; Wan, X.; Chen, Y.; Zhang, Q. J. Mater. Chem. C 2015, 3, 4698.

(44) Liu, S.-Y.; Wu, C.-H.; Li, C.-Z.; Liu, S.-Q.; Wei, K.-H.; Chen, H.-Z.; Jen, A. K. Y. Adv. Sci. 2015, 2, 1500014.

(45) Liu, Y.; Mu, C.; Jiang, K.; Zhao, J.; Li, Y.; Zhang, L.; Li, Z.; Lai, J. Y. L.; Hu, H.; Ma, T.; Hu, R.; Yu, D.; Huang, X.; Tang, B. Z.; Yan, H. Adv. Mater. 2015, 27, 1015.

(46) Shi, Y.; Wu, H. X.; Xue, L.; Li, X. Y. J. Colloid Interface Sci. 2012, 365, 172.

(47) Osswald, P.; Wurthner, F. Chem.-Eur. J. 2007, 13, 7395.

(48) Fimmel, B.; Son, M.; Sung, Y. M.; Grüne, M.; Engels, B.; Kim, D.; Würthner, F. Chem.Eur. J. 2015, 21, 615. $47,5554$.

(49) Huang, Y. W.; Hu, J. C.; Kuang, W. F.; Wei, Z. X.; Faul, C. F. J. Chem. Commun. 2011,

(50) Yan, Q. F.; Zhao, D. H. Org. Lett. 2009, 11, 3426.

(51) de Meijere, A.; Diederich, F. Metal-Catalyzed Cross-Coupling Reactions, 2nd ed.; Wiley-VCH, 2004.

(52) Chinchilla, R.; Najera, C. Chem. Rev. 2007, 107, 874.

(53) Sonogashira, K. J. Organomet. Chem. 2002, 653, 46.

(54) Pandey, P.; Farha, O. K.; Spokoyny, A. M.; Mirkin, C. A.; Kanatzidis, M. G.; Hupp, J. T.; Nguyen, S. T. J. Mater. Chem. 2011, 21, 1700.

(55) Johansson Seechurn, C. C. C.; Kitching, M. O.; Colacot, T. J.; Snieckus, V. Angew. Chem. Int. Ed. 2012, 51, 5062.

(56) Reichert, V. R.; Mathias, L. J. Macromolecules 1994, 27, 7015.

(57) Shen, C.; Yu, H.; Wang, Z. Chem. Commun. 2014, 50, 11238.

(58) Sandstrom, J. Dynamic NMR Spectroscopy, Academic Press, 1982. 
(59) Casarini, D.; Lunazzi, L.; Mazzanti, A. Eur. J. Org. Chem. 2010, 2035.

(60) Gasparro, F. P.; Kolodny, N. H. J. Chem. Ed. 1977, 54, 258.

(61) Eisenberg, D.; Filatov, A. S.; Jackson, E. A.; Rabinovitz, M.; Petrukhina, M. A.; Scott, L. T.; Shenhar, R. J. Org. Chem. 2008, 73, 6073.

(62) Lunazzi, L.; Mancinelli, M.; Mazzanti, A. J. Org. Chem. 2008, 73, 2198.

(63) Schwab, G.; Stern, D.; Stalke, D. J. Org. Chem. 2008, 73, 5242.

(64) Wurthner, F.; Stepanenko, V.; Chen, Z. J.; Saha-Moller, C. R.; Kocher, N.; Stalke, D. J. Org. Chem. 2004, 69, 7933.

(65) Chen, Z. J.; Baumeister, U.; Tschierske, C.; Wurthner, F. Chem.-Eur. J. 2007, 13, 450.

(66) Chen, Z. J.; Debije, M. G.; Debaerdemaeker, T.; Osswald, P.; Wurthner, F. ChemPhysChem 2004, 5, 137. 